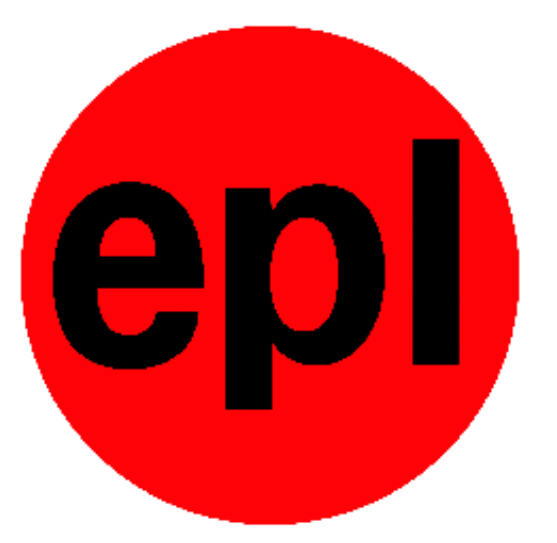

A LETTERS JOURNAL EXPLORING THE FRONTIERS OF PHYSICS

\title{
OFFPRINT
}

\section{Low-pressure gas breakdown in dual-frequency RF electric fields in nitrogen}

\author{
V. Lisovskiy, J.-P. Booth, K. Landry, D. Douai, V. Cassagne \\ and V. YEGORENKOV \\ EPL, 80 (2007) 25001
}

Please visit the new website

www.epljournal.org 


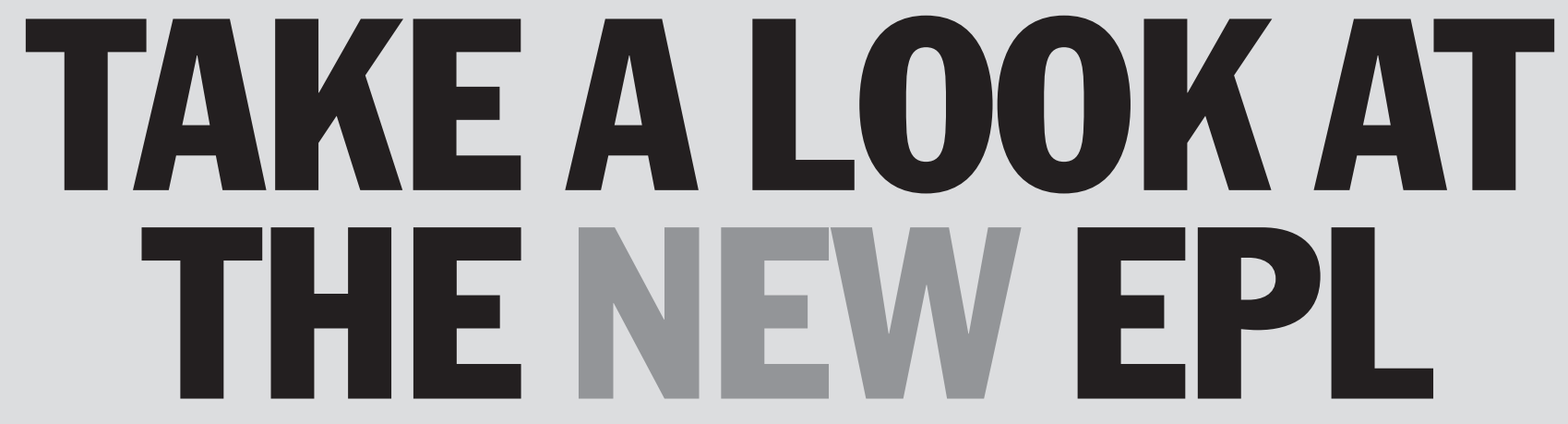

\section{Europhysics Letters (EPL) has a new online home at www.epljournal.org}

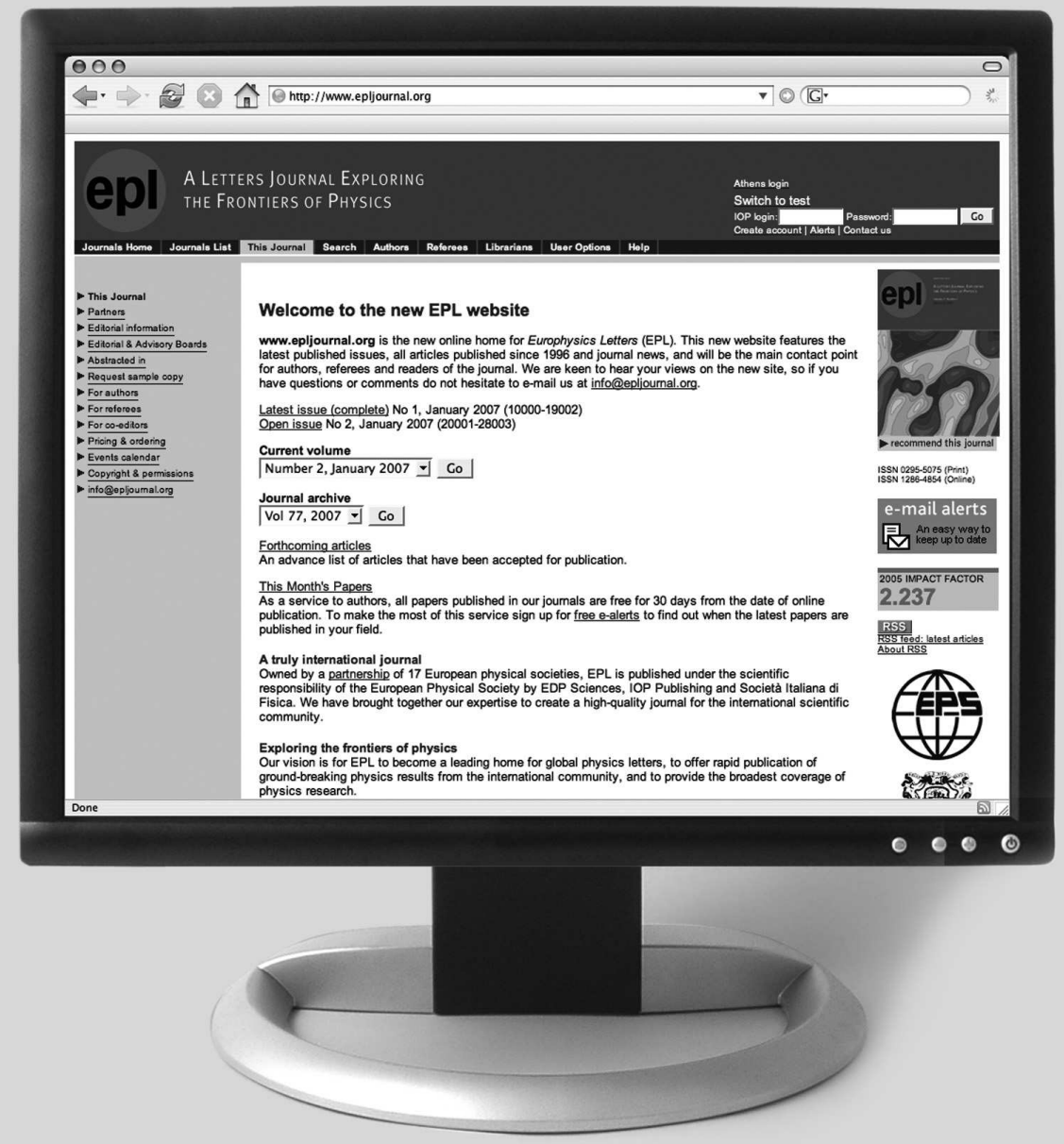

Take a look for the latest journal news and information on:

- reading the latest articles, free!

- receiving free e-mail alerts

- submitting your work to EPL 


\title{
Low-pressure gas breakdown in dual-frequency RF electric fields in nitrogen
}

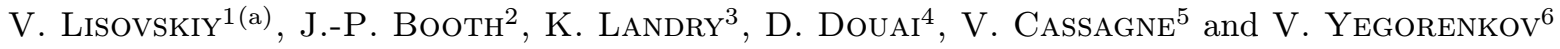 \\ ${ }^{1}$ Department of Physics and Technology, Kharkov National University - Kharkov 6107r, Ukraine \\ ${ }^{2}$ Laboratoire de Physique et Technologie des Plasmas, Ecole Polytechnique - Palaiseau 91128, France \\ ${ }^{3}$ Unaxis Displays Division France SAS - 5 rue Leon Blum, Palaiseau 91120, France \\ ${ }^{4}$ Association Euratom-CEA, Département de Recherches sur la Fusion Contrôlée, CEA Cadarache \\ F-13108 Saint Paul lez Durance Cedex, France \\ ${ }^{5}$ Riber - 31 rue Casimir Périer, 95873 Bezons, France \\ ${ }^{6}$ Department of Physics, Kharkov National University - Kharkov 61077, Ukraine
}

received 17 June 2007; accepted in final form 23 August 2007

published online 17 September 2007

PACS 52.80.Pi - High-frequency and RF discharges

\begin{abstract}
This paper reports the recorded breakdown curves for dual-frequency $(27.12 \mathrm{MHz} / 2 \mathrm{MHz}$ and $13.56 \mathrm{MHz} / 50 \mathrm{~Hz})$ discharges in nitrogen. Applying the LF voltage shifts the $\mathrm{RF}$ breakdown curve to the region of higher voltages and gas pressures, which is associated with the increased loss of charged particles due to the drift in the LF field. At higher LF voltage amplitudes the LF field contributes to gas ionization, the breakdown voltage for the RF discharge decreases and approaches zero when a self-sustained discharge in the LF field ignites. Applying the $\mathrm{RF}$ voltage leads to the decrease in the breakdown LF voltage, possibly due to the decrease of electron losses because of the oscillations in the RF field.
\end{abstract}

Copyright (C) EPLA, 2007

Recently dual-frequency discharges have found broad application in technological processes of etching $\mathrm{SiO}_{2}[1-4]$, $\mathrm{Si}$ and $\mathrm{Si}_{3} \mathrm{~N}_{4}$ [3], depositing $\mathrm{SiO}_{2}, \mathrm{Si}_{3} \mathrm{~N}_{4}[5,6]$. In a conventional $\mathrm{RF}$ capacitive discharge burning at a fixed frequency $f$ in a discharge chamber with a gap $L$ between the electrodes, at a fixed gas pressure $p$ and a certain value of $\mathrm{RF}$ power, the flow of ions to the electrodes and ion energy remain fixed. It is impossible to control these two quantities in a conventional single-frequency $\mathrm{RF}$ discharge separately. The problem of separately controlling the ion flow to the electrodes and ion energy is easily solved in the RF dual-frequency discharges, as the high-frequency electric field provides dense plasma while the low-frequency one increases the voltage drop across the near-electrode sheath, and as a result, accelerates ions to higher energy.

Therefore an increased interest to studying processes in dual-frequency discharges is observed [7-16]. At the same time, the gas breakdown in dual-frequency RF discharge remains almost unknown. Whereas the gas breakdown in a single-frequency RF discharge is studied in much detail [17-19], the ignition of a dual-frequency RF discharge still remains to be unexplored.

\footnotetext{
(a) E-mail: lisovskiy@yahoo.com
}

This paper studies experimentally the ignition of a dual-frequency $(27.12 \mathrm{MHz} / 2 \mathrm{MHz}$ and $13.56 \mathrm{MHz} / 50 \mathrm{~Hz})$ discharge in nitrogen. The application of a LF voltage $(2 \mathrm{MHz}$ or $50 \mathrm{~Hz}$ ) is shown to increase the RF breakdown voltage, the RF breakdown curve being also shifted to the region of higher gas pressure. At high LF voltage values close to the breakdown potential of the LF discharge, the $\mathrm{RF}$ breakdown voltage decreases to zero. Breakdown curves of the $13.56 \mathrm{MHz} / 50 \mathrm{~Hz}$ dual-frequency discharge are similar to the breakdown curves of the RF/DC combined discharge qualitatively, however considerable quantitative deviations in the $\mathrm{RF}$ breakdown voltage values are observed.

Experiments were performed in two different set-ups. At the first set-up the RF discharge was ignited in nitrogen over the pressure range $p \approx 0.03-6$ Torr. RF voltages with the frequencies of $27.12 \mathrm{MHz}(\mathrm{RF})$ and $2 \mathrm{MHz}(\mathrm{LF})$ were fed to the same powered electrode whereas the other one was grounded. The inter-electrode gap was $20.4 \mathrm{~mm}$. The electrodes (143 $\mathrm{mm}$ in diameter) were located inside a fused silica tube with an inner diameter of $145 \mathrm{~mm}$. The gas was supplied through small orifices in the powered electrode and then pumped out via the gap between the second electrode and the wall of the fused silica tube. 


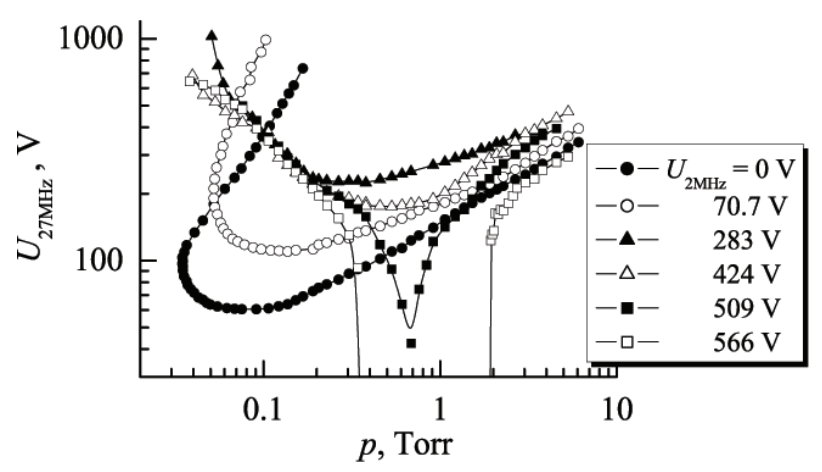

Fig. 1: RF breakdown curves for $27.12 \mathrm{MHz}$ at various fixed values of the $\mathrm{LF}$ voltage with the frequency of $2 \mathrm{MHz}$.

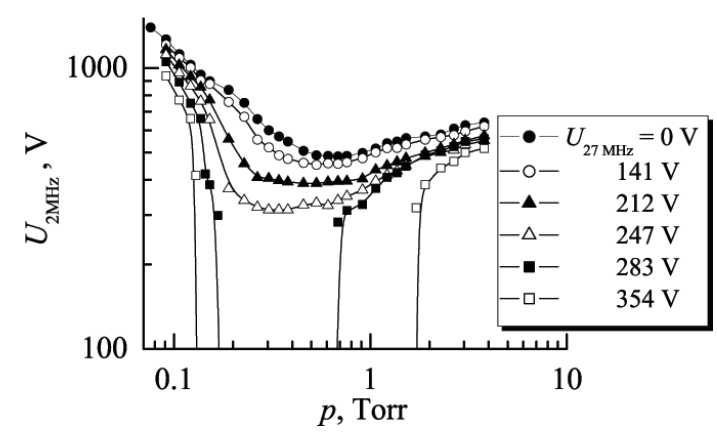

Fig. 2: $\mathrm{LF}$ breakdown curves for $2 \mathrm{MHz}$ at various fixed values of the RF voltage with the frequency of $27.12 \mathrm{MHz}$.

The gas pressure was monitored with 10 and 1000 Torr capacitive manometers (MKS Instruments). The gas flow was fixed with a mass flow controller to $5 \mathrm{sccm}$, and the pressure regulated by throttling the outlet to the pump. The $\mathrm{RF}$ voltage was measured with an RF current-voltage probe (Advanced Energy Z'SCAN).

In another research set-up the RF discharge was sustained between two parallel-plate stainless-steel electrodes of $10 \mathrm{~cm}$ in diameter and a discharge gap of $3 \mathrm{~cm}$ width. RF voltage with the frequency $13.56 \mathrm{MHz}$ (RF) and LF voltage with the frequency $50 \mathrm{~Hz}$ (LF) were fed to the same powered electrode whereas the other one was grounded. The gas delivery and exhaust were accomplished through groups of small holes in one of the electrodes.

Figure 1 shows the RF breakdown curves for $27 \mathrm{MHz}$ ( $\mathrm{RF}$ breakdown voltage with the frequency of $27 \mathrm{MHz}$ against gas pressure with fixed values of the LF voltage with the frequency of $2 \mathrm{MHz}$ ). And fig. 2 shows the LF breakdown curves for $2 \mathrm{MHz}$ (LF breakdown voltage with the frequency of $2 \mathrm{MHz}$ against gas pressure with fixed values of the $R F$ voltage with the frequency of $27 \mathrm{MHz}$ ). In general an RF breakdown curve may possess the multipactor, Paschen and diffusion-drift branches [17]. The RF breakdown curve for $U_{2 \mathrm{MHz}}=0$ within the total pressure range we studied consists of a single diffusion-drift branch (fig. 1). The LF breakdown curve for $U_{27 \mathrm{MHz}}=0$ possesses

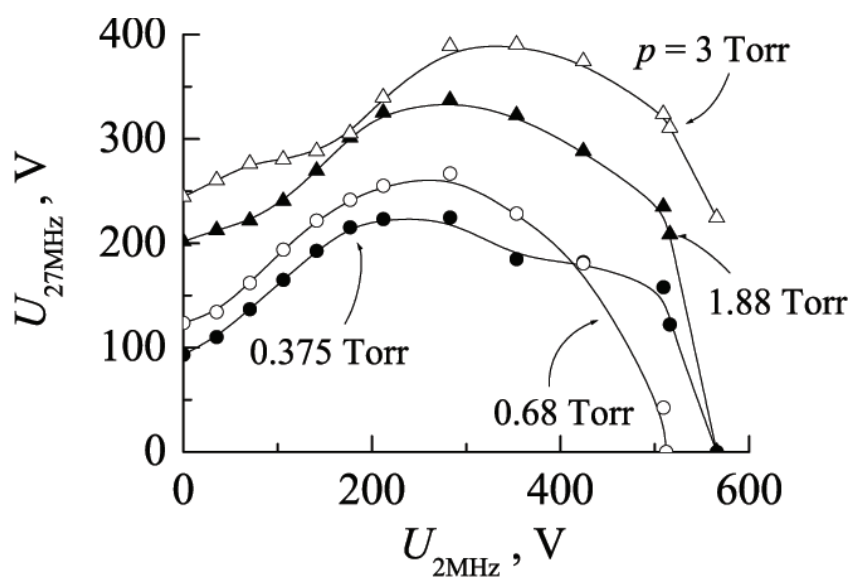

Fig. 3: RF breakdown voltage for $27.12 \mathrm{MHz}$ against the $\mathrm{LF}$ voltage with the frequency of $2 \mathrm{MHz}$ at various fixed values of nitrogen pressure.

only the Paschen branch (fig. 2), whereas the diffusiondrift branch may be observed only at higher gas pressure. Applying the LF voltage with the frequency of $2 \mathrm{MHz}$ $U_{2 \mathrm{MHz}}<300 \mathrm{~V}$ shifts the RF breakdown curve for $27 \mathrm{MHz}$ to the region of higher voltages and gas pressures (see figs. 1 and 3). It may be associated with the increased loss of electrons due to the drift in the LF field. At the same time, the section of the multi-valued dependence of the RF breakdown voltage with the frequency $27 \mathrm{MHz}$ against gas pressure, observed at low pressure values, narrows and at $U_{2 \mathrm{MHz}}>250 \mathrm{~V}$ disappears, yielding $\mathrm{RF}$ breakdown curves possessing the U-like shape. At higher $U_{2 \mathrm{MHz}}$ amplitudes the LF field contributes to gas ionization via electron impact. Besides, positive ions oscillating in the enhanced LF field can collide with the electrode surface and produce secondary electrons. At $430 \mathrm{~V}<U_{2 \mathrm{MHz}}<510 \mathrm{~V}$ a sharp minimum appears in $\mathrm{RF}$ breakdown curves for $27 \mathrm{MHz}$ at the pressure value of $p \approx 0.7$ Torr. The minimum in the $\mathrm{LF}$ breakdown curve for $2 \mathrm{MHz}$ appears at the same pressure value (see curve for $U_{27 \mathrm{MHz}}=0$ ). The RF breakdown voltage for $U_{2 \mathrm{MHz}}>300 \mathrm{~V}$ decreases and approaches zero when a self-sustained LF discharge ignites.

Applying the RF voltage with the $27 \mathrm{MHz}$ frequency leads to the decrease in the LF breakdown voltage $U_{2} \mathrm{MHz}$ (see figs. 2 and 4). This may be caused by the decrease of electron losses because of the oscillations in the RF electric field. Besides, the RF field makes a contribution to the ionization of gas molecules. Increasing the RF voltage leads to the shift of the LF breakdown curves to the region of lower gas pressure and LF voltage.

On recording the RF breakdown curves we first fixed gas pressure and applied a chosen LF voltage, and then increased the $\mathrm{RF}$ voltage up to the moment of gas breakdown. However, for studying the effect of the RF voltage on the LF breakdown curves we first applied the LF voltage $U_{2 \mathrm{MHz}} \approx 200 \mathrm{~V}$, then we fed the chosen value of the $R F$ voltage and, only after that, increased the LF voltage up to the moment of gas breakdown. If 


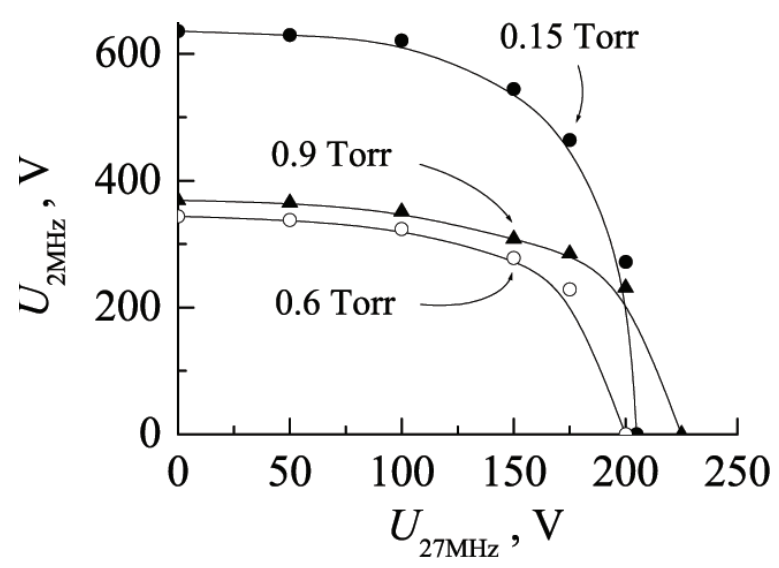

Fig. 4: $\mathrm{LF}$ breakdown voltage for $2 \mathrm{MHz}$ against the $\mathrm{RF}$ voltage with the frequency of $27.12 \mathrm{MHz}$ at various fixed values of nitrogen pressure.

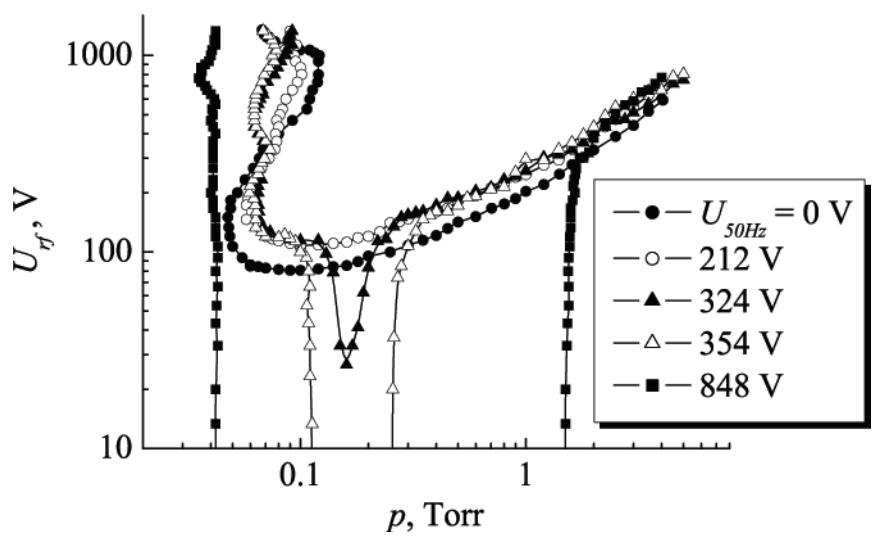

Fig. 5: RF breakdown curves for $13.56 \mathrm{MHz}$ at various fixed values of the $\mathrm{LF}$ voltage with the frequency of $50 \mathrm{~Hz}$.

the LF voltage is not applied first, but the RF voltage is fed at once, then we obtain the ignition of the RF discharge at rather low RF voltages. For example, at $p=1$ Torr for igniting the RF discharge the application of $U_{27 \mathrm{MHz}}=154 \mathrm{~V}$ is required. At the same time, it is clear from fig. 2, that such low RF voltage almost does not affect the ignition of the LF discharge. Therefore we first prevented the ignition of the self-sustained RF discharge via applying the $L F$ voltage, and only then applied the $R F$ voltage, to be able to study the effect of high RF voltages on the LF breakdown.

Now consider our results for dual-frequency $13.56 \mathrm{MHz} / 50 \mathrm{~Hz}$ discharges, obtained in the second set-up. Applying the LF voltage of $50 \mathrm{~Hz} U_{50 \mathrm{~Hz}}$ first shifts the RF breakdown curve for $13.56 \mathrm{MHz}$ to the region of higher gas pressure and $\mathrm{RF}$ voltage (see fig. 5). On increasing the LF voltage further, two features are observed in the RF breakdown curve. When the LF voltage applied is close to the minimum breakdown voltage of the LF discharge, a sharply expressed minimum is observed in the RF breakdown curve. At higher LF voltages a self-sustained LF discharge is ignited, therefore

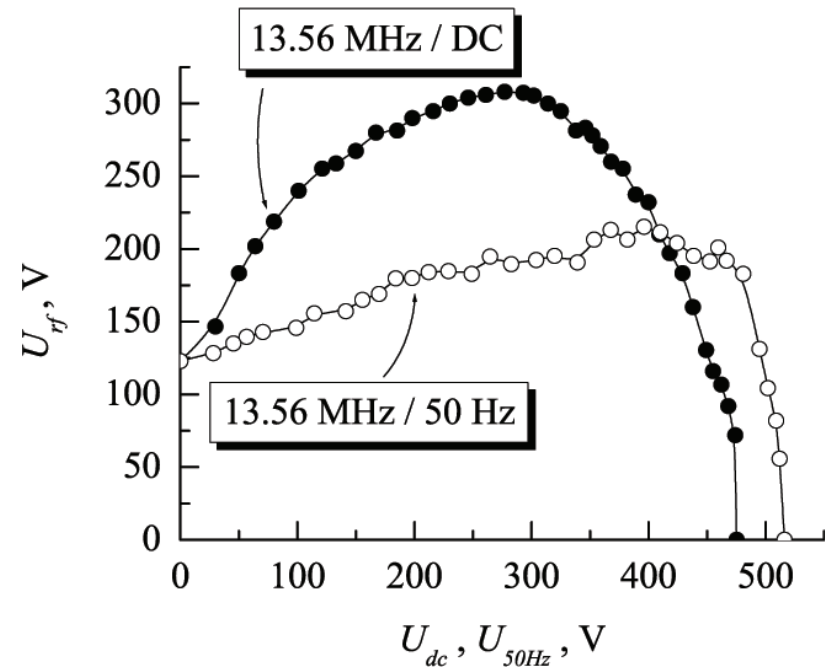

Fig. 6: RF breakdown voltage for $13.56 \mathrm{MHz}$ against the $\mathrm{DC}$ and $\mathrm{LF}$ voltage with the frequency of $50 \mathrm{~Hz}$ at nitrogen pressure 0.5 Torr.

the RF breakdown voltage equals zero (see fig. 6). At LF voltages $U_{50 \mathrm{~Hz}}>200 \mathrm{~V}$ an additional region of multivalued dependence of the $R F$ breakdown voltage against pressure is observed. With the LF voltage increasing, this multi-valued region is shifted to higher values of RF voltage and lower values of gas pressure. Such a multi-valued region was observed earlier in the breakdown curves of the longitudinal combined (RF/DC) discharge [20]. The paper [20] performed a detailed analysis of the observed phenomenon, so we will not pay attention to it here.

Despite the qualitative similarity between the shape of the RF breakdown curves obtained with application of $50 \mathrm{~Hz}$ and $\mathrm{DC}$ voltages, the RF breakdown voltage values exhibit remarkable quantitative difference. Figure 6 shows $\mathrm{RF}$ voltages required for the ignition of the combined $(13.56 \mathrm{MHz} / \mathrm{DC})$ and dual-frequency $(13.56 \mathrm{MHz} / 50 \mathrm{~Hz})$ discharges we registered with the nitrogen pressure fixed. Both curves possess a growing section which after attaining a maximum exhibits a decrease of the $\mathrm{RF}$ breakdown voltage. However, the application of the $50 \mathrm{~Hz}$ voltage involves a much less increase of the $\mathrm{RF}$ breakdown voltage than the application of the $\mathrm{DC}$ voltage. It may be attributed to uniformly increasing loss of charged particles due to the drift in the DC field associated with the application of the DC voltage. The LF voltage is of a sinusoidal shape with a period $T_{50 \mathrm{~Hz}} \gg T_{13 \mathrm{MHz}}$. Perhaps the dual-frequency $(13.56 \mathrm{MHz} / 50 \mathrm{~Hz})$ discharge is ignited during the time period when the instantaneous LF voltage is small. Here the loss of charged particles due to the drift in the LF field is smaller, and therefore the ignition of the dual-frequency discharge occurs at smaller RF voltages than the ignition of the combined $13.56 \mathrm{MHz} / \mathrm{DC}$ discharge.

Thus, this paper presents the breakdown curves of the dual-frequency discharges $(27.12 \mathrm{MHz} / 2 \mathrm{MHz}$ and $13.56 \mathrm{MHz} / 50 \mathrm{~Hz}$ ) we recorded in nitrogen. The effects 
of the LF voltage on the RF breakdown curves, as well as the effects of the RF voltage on the LF breakdown curves were studied. At small LF voltages applied to the $\mathrm{RF}$ discharge to be ignited, the loss of charged particles to the electrodes increases, thus increasing the $\mathrm{RF}$ breakdown voltage. At large LF voltages contributing to the ionization of gas molecules via electron impact, the $\mathrm{RF}$ breakdown voltage approaches a maximum and then decreases down to zero. Application of the RF voltage always makes the LF discharge ignition easier.

$$
* * *
$$

The authors express their gratitude to the UNAXIS France - Displays division, Palaiseau, France for their financial support and for the equipment used in this study.

\section{REFERENCES}

[1] Goto H. H., Lowe H. D. and Онмі T., J. Vac. Sci. Technol. A, 10 (1992) 3048.

[2] Tsai W., Mueller G., Lindquist R., Frazier B. and VAhedi V., J. Vac. Sci. Technol. B, 14 (1996) 3276.

[3] Matsui M., Tatsumi T. and Sekine M., J. Vac. Sci. Technol. A, 19 (2001) 2089.

[4] Fracassi F., D'Agostino R., Fornelli E., Illuzzi F. and Shirafuji T., J. Vac. Sci. Technol. A, 21 (2003) 638.

[5] Suchaneck G., Guenther M., Sorber J., Gerlach G., Arndt K.-F. and Wolf B., Appl. Phys. A, 78 (2004) 695.
[6] Cianci E., Schina A., Minotti A., Quaresima S. and Foglietti V., Sens. Actuators A: Phys., 127 (2006) 80.

[7] Makabe T. and Maeshige K., Appl. Surf. Sci., 192 (2002) 176.

[8] Robiche J., Boyle P. C., Turner M. M. and Ellingboe A. R., J. Phys. D, 36 (2003) 1810.

[9] Franklin R. N., J. Phys. D, 36 (2003) 2660.

[10] Wakayama G. and Nanbu K., IEEE Trans. Plasma Sci., 31 (2003) 638.

[11] Kim H. C., Lee J. K. and Shon J. W., Phys. Plasmas, 10 (2003) 4545.

[12] Boyle P. C., Robiche J. and Turner M. M., J. Phys. $D, 37$ (2004) 1451.

[13] Georgieva V., Bogaerts A. and Gijbels R., Phys. Rev. E, 69 (2004) 026406.

[14] Salabas A. and Brinkmann R. P., Plasma Sources Sci. Technol., 14 (2005) S53.

[15] Barnat E. V., Miller P. A., Hebner G. A., Paterson A. M., Panagopoulos T., Hammond E. and Holland J., Plasma Sources Sci. Technol., 16 (2007) 330.

[16] Turner M. M. and Chabert P., Plasma Sources Sci. Technol., 16 (2007) 364.

[17] Lisovskiy V. A. and Yegorenkov V. D., J. Phys. D, 31 (1998) 3349.

[18] Lisovskiy V., Martins S., Landry K., Douai D., Booth J.-P., Cassagne V. and Yegorenkov V., Phys. Plasmas, 12 (2005) 093505.

[19] Lisovskiy V., Booth J.-P., Landry K., Douai D., Cassagne V. and Yegorenkov V., J. Phys. D, 39 (2006) 660.

[20] Lisovsky V. A. and Yegorenkov V. D., J. Phys. D, 27 (1994) 2340. 